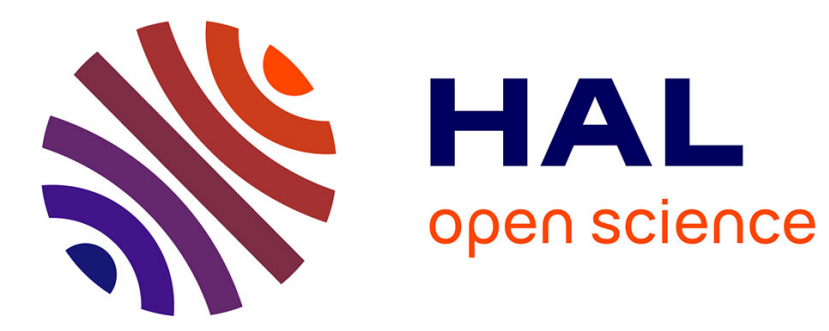

\title{
On frequency-independent horn antenna design for plasma positioning reflectometers, from simulation to prototype testing
}

\author{
J Lips, Stéphane Heuraux, C Lechte, B Plaum
}

\section{- To cite this version:}

J Lips, Stéphane Heuraux, C Lechte, B Plaum. On frequency-independent horn antenna design for plasma positioning reflectometers, from simulation to prototype testing. Journal of Instrumentation, 2021, JINST, 16 (7), pp.P07040. 10.1088/1748-0221/16/07/P07040 hal-03272505

\section{HAL Id: hal-03272505 \\ https://hal.univ-lorraine.fr/hal-03272505}

Submitted on 28 Jun 2021

HAL is a multi-disciplinary open access archive for the deposit and dissemination of scientific research documents, whether they are published or not. The documents may come from teaching and research institutions in France or abroad, or from public or private research centers.
L'archive ouverte pluridisciplinaire HAL, est destinée au dépôt et à la diffusion de documents scientifiques de niveau recherche, publiés ou non, émanant des établissements d'enseignement et de recherche français ou étrangers, des laboratoires publics ou privés. 


\title{
On frequency-independent horn antenna design for plasma positioning reflectometers, from simulation to prototype testing
}

\author{
J. Lips, ${ }^{a, 1}$ S. Heuraux, ${ }^{b}$ C. Lechte ${ }^{c}$ and B. Plaum ${ }^{c}$ \\ ${ }^{a}$ University of Stuttgart, Institute for Combustion and Power Plant Technology (IFK), \\ D-70569 Stuttgart, Germany \\ ${ }^{b}$ Université de Lorraine, Institut Jean Lamour, \\ F-5401 Nancy, France \\ ${ }^{c}$ University of Stuttgart, Institute of Interfacial Process Engineering and Plasma Technology (IGVP), \\ D-70569 Stuttgart, Germany
}

E-mail: johannes.lips@ifk.uni-stuttgart.de

\begin{abstract}
Using a new framework for horn antenna optimization, a first frequency-independent horn antenna for a plasma positioning reflectometer (PPR) is designed. This hardware optimization is a new approach aiming on lowering the computational complexity of PPR interpretative models able to extract profiles and fluctuations of electron density and temperature from reflectometry measurements. PPR measurements are a strong candidate to provide real-time feedback for plasma position control in long-discharge fusion tokamaks such as ITER and DEMO, which requires fast interpretative models. Reducing the computational complexity of these models is therefore critical. In this paper, ray tracing simulations in the poloidal plane are used to compare different radiation patterns and evaluate them on multiple performance criteria. An antenna shape leading to a frequency-independent far-field radiation diagram is found with the PROFUSION optimizer and a prototype of this antenna is tested against an unoptimized conical reference antenna. The ray tracing performance criteria indicate that fundamental Gaussian frequency-independent antennas perform well, albeit in general worse than same-sized frequency-dependent antennas. Furthermore, it is seen that the pyramidal ITER gap 6 antenna performs well given the spatial constraints and that more performant antennas could be used, when more space was available. The prototype testing reveals challenges in obtaining frequencyindependent characteristics within a tokamak blanket environment, which is additionally complicated by misalignment risks.
\end{abstract}

KeYwords: Microwave Antennas; Nuclear instruments and methods for hot plasma diagnostics

\footnotetext{
${ }^{1}$ Corresponding author.
} 


\section{Contents}

1 Introduction 1

2 Ray tracing simulations 2

2.1 Context 2

2.2 Code description 3

2.3 Simulated radiation patterns and results 5

$\begin{array}{lll}3 & \text { Radiation pattern choice } & 7\end{array}$

4 Antenna design $\quad 8$

4.1 Methodology 8

$\begin{array}{lll}4.2 & \text { Optimization procedure } & 8\end{array}$

$\begin{array}{lll}4.3 & \text { Higher-order mode analysis } & 9\end{array}$

5 Experimental prototype testing 10

$\begin{array}{lll}5.1 \text { Measurement setup } & 10\end{array}$

$\begin{array}{ll}5.2 \text { Radiation diagram analysis } & 11\end{array}$

6 Conclusions 14

\section{Introduction}

Reflectometry is known to be a versatile tool able to give access to macroscopic plasma parameters (density profile, plasma velocity, radial electric field) or to density perturbations, which are associ-ated to turbulence [1], MagnetoHydroDynamic activities [2], mode excitations by fast particles [3], and more $[4,5]$. However, to access all of this information, it is necessary to have interpretative models adapted to the different kinds of microwave systems. Most of the reflectometers work with the possibility to change the probing frequency in a continuous way (ultra-fast sweep frequency [6]), or step-by-step (frequency hopping system [7], Doppler reflectometry [8]). To measure density profiles, frequently pyramidal [6,9] or conical [10] horns are used with frequency sweeps. Often the interpretative models used to rebuild the density profile ignore some effects such as the Doppler shift induced to the phase center position [11] or neglect radiation pattern changes during the fre- quency sweep. This can be justified in some cases but requires (a) to interpret the measured signals independent of their amplitude and (b) the wavefront of the received wave to be approximated as a plane wave using WKB approximation [12, 13]. However, these kinds of approximations fail when the system is operated close to the cut-off layers. An interpretative model with a transfer function that links probing frequency to the cut-off surface position and phase measurements to the wave- number spectrum of the turbulence is able to extract both density profile and density fluctuation 
measurements but becomes computationally complex [14]. A possible way to reduce the complex-ity of this type of model is to build hardware components for are 'frequency-independent' within the frequency range of the diagnostic, which is defined here as having a frequency-independent divergence angle. Such a system together with a simplified interpretative model would be relevant both in research as well as in operation of a plasma positioning reflectometer (PPR) providing realtime feedback for the plasma positioning control system of an operational tokamak $[15,16]$. The aim of this paper is to present a possible path to build a horn antenna having such emission properties. Section 2 presents a ray tracing simulation code, based on the current ITER plasma positioning reflectometer environment. The simulations assess the performance of antennas with various radiation characteristics and their resilience against density fluctuations. This leads to the choice of an optimal radiation pattern respecting the ITER plasma positioning reflectometer spatial constraints, presented in Section 3. In section 4, the shape of a cylindrical horn antenna is optimized to result in this radiation pattern characteristics. In section 5 a prototype was tested in different conditions, including the case of the expected set-up of the in-vessel ITER plasma positioning reflectometers. Additionally, the impact of the antenna placing and relative alignment with the surrounding blanket modules was determined, as well as the effects of higher-order input modes. Section 6 presents the conclusions and recommendations for further work.

\section{Ray tracing simulations}

\subsection{Context}

In blanket-equipped tokamaks, plasma density fluctuations and multi-reflections can strongly impact the performance of PPR $[17,18]$. In order to compare the performance of antennas with different radiation characteristics in such an environment, parametric studies were done with a ray tracing code in Python. As environment, the nominal setup of the ITER gap 6 PPR antenna and the surrounding blanket modules, studied by P. Valera, J.H. Belo and A. Silva $[19,20]$ is used. The poloidal section of this environment is drawn in Figure 1. For all simulations, the plasma minor radius $a=2 \mathrm{~m}$ and central plasma density $n_{0}=10^{20} \mathrm{~m}^{-3}$ are used with a quadratic density profile.

$$
n=n_{0}\left(1-r^{2} / a^{2}\right)=n_{0}\left(1-\left(x^{2}+z^{2}\right) / a^{2}\right)
$$

The probing frequencies are limited to the range of $30 \mathrm{GHz}$ to $60 \mathrm{GHz}$. The corresponding O-mode cut-off layers and densities are found in Table 1.

Knowing that full-wave simulations for the pyramidal horn reflectometer setup in ITER [17] and DEMO $[9,21]$ already have been done, and that parametric studies require a large number of simulations (specifically parameter changes in probing frequency, level of turbulence and radiation pattern will be considered), computationally cheap ray tracing [22] with a WKB-based numeric solver in the plasma and an analytic solver outside of the plasma was chosen as simulation approach. Gaussian beam tracing [23], taking into account beam divergence, was considered but rejected, because of a larger computation time arising from the numeric solver in the complete domain, which would be necessary for the correct simulation of divergence, reflections on metallic surfaces and the detection of the rays received by the antenna. All these aspects are simplified with the suggested ray tracing approach. 


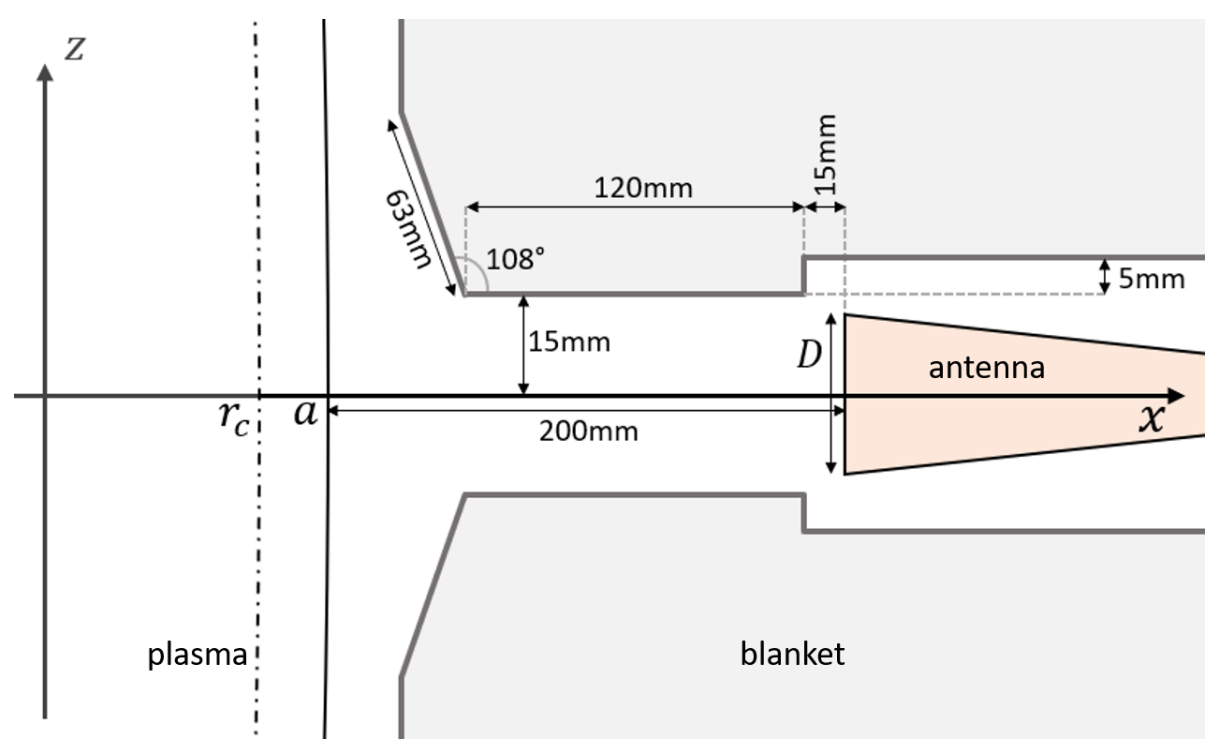

Figure 1: The geometry of the simulation environment (poloidal view) based on the ITER gap 6 setup [19, 20], with $D$ the antenna aperture, $a$ the plasma minor radius and $r_{c}$ the cut-off layer.

\subsection{Code description}

The simulated environment includes turbulent plasma, metallic blankets and the PPR antenna. Outside the plasma, vacuum propagation is assumed with perfect specular reflection when a ray hits a blanket. A numerical solver is used inside the plasma to solve the O-mode dispersion relation using the WKB approximation [22, Chapter 3]. This means that the condition

$$
L>k_{0}^{-1}
$$

needs to be fulfilled [24]. The density gradient length $L$ at the cut-off $n_{c}\left(r_{c}\right)$ is defined as [25]

$$
L=-n_{c} \cdot\left(\left.\frac{\mathrm{d} n}{\mathrm{~d} r}\right|_{r_{c}}\right)^{-1}
$$

Applied to the quadratic density profile (Eq. 2.1), this becomes

$$
L=\frac{n_{c}}{n_{0}} \frac{a^{2}}{2 r_{c}}
$$

It can be seen in Table 1 that the WKB condition (Eq. 2.2) is satisfied for all probing frequencies.

The code considers forward scattering, which is known to have major effects on spreading the probing beam associated with the multi-reflections [26] and can be described in the WKB framework compatible with the used ray tracing. MHD effects, corresponding with larger structures, and Bragg backscattering, corresponding with smaller structures, are not included. The corresponding density fluctuation model $\delta n$ is assumed homogeneous and is built from a superposition of density

fluctuations, each with a certain $\vec{k}_{\text {fluc }}=k_{\tilde{x}} \vec{e}_{\tilde{x}}+k_{\tilde{z}} \vec{e}_{\tilde{z}}\left(k_{\text {fluc }}=\forall k_{z}\right)$, in whick $\overline{\left.k_{\tilde{x}}^{2} \tilde{x}, \tilde{z}\right)^{2}}$ are coordinates used for a slab model description of the plasma. The resulting density perturbation model is given by [27]:

$$
\delta n_{\text {turb }}(\tilde{x}, \tilde{z})=\sum_{i=i_{m}}^{i_{M}} \sum_{j=j_{m}}^{j_{M}} A(i, j) \cos \left[k_{\text {fluc }, \tilde{x}}(i) \tilde{x}+k_{\text {fluc }, \tilde{z}}(j) \tilde{z}+\phi(i, j)\right]
$$


Table 1: Cut-off layer $r_{c}$, cut-off density $n_{c}$, gradient length $L$ and inverse wavenumber $k_{0}^{-1}$ at the frequency limits

\begin{tabular}{|l|ll|}
\hline Frequency & $30 \mathrm{GHz}$ & $60 \mathrm{GHz}$ \\
\hline$r_{c}$ & $1.885 \mathrm{~m}$ & $1.487 \mathrm{~m}$ \\
$n_{c}$ & $1.12 \cdot 10^{19} \mathrm{~m}^{-3}$ & $4.46 \cdot 10^{19} \mathrm{~m}^{-3}$ \\
$L$ & $10 \mathrm{~mm}$ & $600 \mathrm{~mm}$ \\
$k_{0}^{-1}$ & $1.6 \mathrm{~mm}$ & $0.8 \mathrm{~mm}$ \\
\hline
\end{tabular}

with $(i, j)$ summing over all wavenumbers within the range corresponding to dominant forward scattering, $A(i, j)$ is the amplitude spectrum, and $\phi(i, j)$ consisting of random generated phases for each $(i, j)$. In the ray tracing code, a polar turbulence model based on the slab model is used (under transformation $\tilde{x}=r, \tilde{z}=\theta r$, which is valid when the spatial dimensions are large compared to the wavelengths associated with the fluctuations in the plasma and $\tilde{z} \ll a$ ).

To determine the range of wavenumbers and $A(i, j)$, the methodology of F. da Silva et al for a Doppler reflectometry study [27] was followed. An upper limit for the wavenumber range in which forward scattering is dominant is [25]:

$$
k_{\text {fluc }} \leq 2 k_{A}
$$

with the Airy wavenumber $k_{A}$ :

$$
k_{A}=0.63 k_{0}^{2 / 3} L^{-1 / 3}
$$

To allow for a fair comparison between simulations at different probing frequencies, $k_{\text {up }}=2 k_{A}$ should be constant; $k_{A}(30 \mathrm{GHz})=87.01 \mathrm{rad} / \mathrm{m}$ is chosen. A lower limit to the fluctuation wavenum- bers was chosen at $k_{\text {low }}=50 \mathrm{rad} / \mathrm{m}$, leaving out MHD turbulence such as tearing modes and mag-netic islands which have lower fluctuation wavenumbers. The resulting turbulence model has wave numbers in the range:

$$
50 \mathrm{rad} / \mathrm{m} \leq k_{\text {fluc }} \leq 174 \mathrm{rad} / \mathrm{m}
$$

For the amplitude $A(i, j)$ associated with each considered fluctuation $(i, j)$, a cubic decay [28] is used:

$$
A\left(k_{\text {fluc }}\right)=\frac{B}{\left(k_{\text {fluc }}-C\right)^{3}}
$$

in which $B$ and $C$ are constants which adjust the shape of the decay and the turbulence level. This results in a model which is in close agreement (both in used range for the fluctuations as in amplitude) with the forward scattering model used by F. da Silva [27]. In edge regions where the sum of the quadratic main density profile and the density fluctuation models would be negative, density is set to zero.

The plasma is considered stationary, which is justified as the current hardware permits prob- ing times much smaller than the smallest timescale for changes in the described plasma density fluctuations [6]. Rays are launched from a focal point behind the aperture according to the far field radiation ${ }_{142}$ pattern [29]. When the rays return to the antenna, a phase front reconstruction is made using adjacent rays, from which the returned power to the antenna over time and the time of flight can be retrieved. 


\subsection{Simulated radiation patterns and results}

Amongst the simulated patterns are the diagram of the currently suggested pyramidal horn antenna for ITER gap 6 [30] and several cylindrical fundamental Gaussians as well as Gaussians with higher-order modes. Of the Gaussian radiation patterns, some were frequency independent, with beam waist $w_{0}$ proportional to the probing wavelength $\lambda$

$$
w_{0} \propto \lambda
$$

Other patterns had a fixed beam waist, resulting in standard frequency-dependent radiation diagrams. Different antenna apertures were also simulated. The list of simulated radiation patterns is given in Table 2. For each radiation pattern, simulations were done at five equidistant frequencies in the range 30 to $60 \mathrm{GHz}$ for $0 \%, 0.25 \%, 1 \%, 3 \%, 5 \%$ and $10 \%$ RMS turbulence relative to the cut-off density at $30 \mathrm{GHz}$. For each of these parameter sets, an ensemble of 400 simulations (each with different, statistically independent phase matrix $\phi$ ) was used to allow for statistical analysis. Independent of the simulated radiation pattern and the probing frequency, it was found that for very low turbulence $(0.25 \%)$ only rays from the central region of the radiation diagram had a chance of returning to the antenna. With increasing turbulence, two phenomena were observed: the central region of the radiation diagram contributing to returned power widened gradually (due to an increased chance on the turbulent plasma deflecting a ray previously hitting the blanket towards ${ }_{161}$ the antenna aperture), while the probability for a ray in this region to contribute got lower (due to an increased chance on the turbulent plasma deflecting a ray previously hitting the aperture towards the blankets). The combination of these effects leads to a maximum power return at approximately $3 \%$ turbulence, depicted on Figure 2: up to $3 \%$ of turbulence, the first effect is dominant; at higher turbulence, the second effect becomes dominant. This phenomenon is also described by [27, 31]. In [27], full-wave simulations also found a maximum amplitude of the return signal around $3 \%$ turbulence, agreeing with the results that are presented here.

On other points, the results varied depending on the pattern. Multiple methods were used to quantify these differences. The average values (averaged over all frequencies and all turbulence from $0.25 \%$ to $10 \%$ ) of the following performance indicators can be found in Table 2:

- Fraction of Invalid Runs (IR): the fraction of the ensemble for which no valid time of flight could be determined due to a lack of useful signal received.

- Antenna Coupling (AC): the percentage of power launched in the plane returning to the antenna.

- Share of rays with multi-reflection trajectories in AC (MR): the fraction of the power returning to the antenna coming from rays which underwent multi-reflections in their trajectory, meaning that they undergo at least one specular reflection on the blankets.

On these criteria, the $\mathrm{TEM}_{00} / \mathrm{TEM}_{10}$ superpositions score badly, with notably higher fractions of invalid runs and lower antenna coupling (Table 2). From comparison of the results between G14F and G28F or G14L, G24L and G28L, it is seen that an increase in aperture (and accompanying larger beam waist) is beneficial for the performance of the antenna: the number of invalid runs drops significantly, the antenna coupling goes up and the fraction of MR-rays contributing to the receiver 


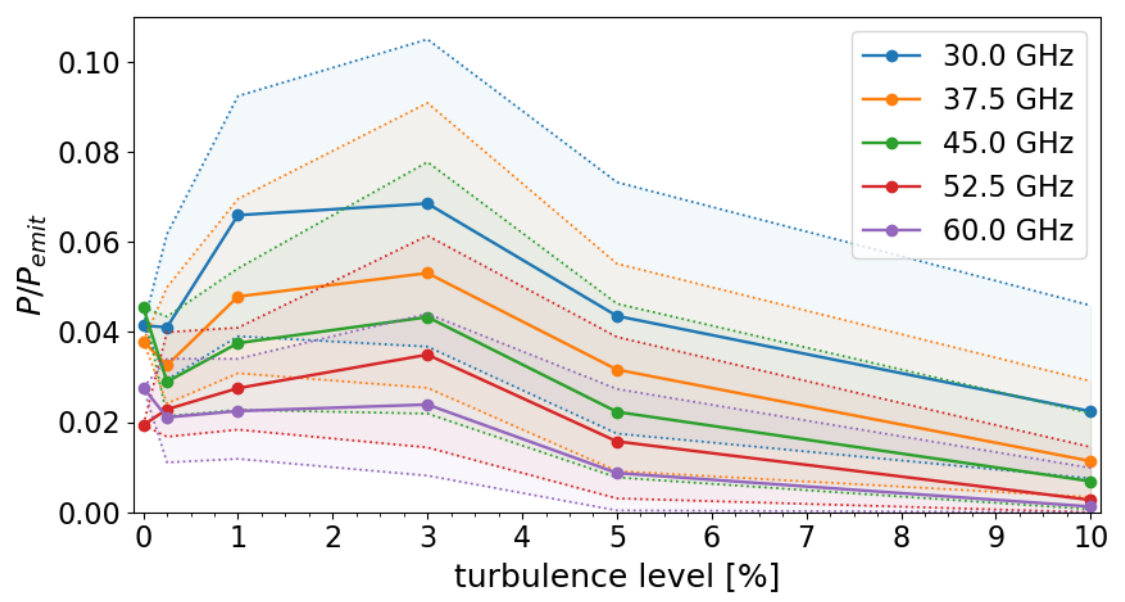

Figure 2: Median cumulative returned power and interquartile range (shaded area) in the poloidal plane at simulation end time for a pyramidal horn radiation pattern (PYR) at various frequencies and amounts of turbulence. It can be seen that the total received power reaches a maximum at $\approx 3 \%$ turbulence, in agreement with [27]

signal lowers drastically. From comparison between G14F with G14L and G28F with G28L, it is clear that with the used $w_{0} / \lambda$-relations, frequency-independent radiation patterns have worse performance than their similar-sized frequency-dependent equivalents when it comes to antenna coupling and share of multi-reflections in the received signal. It is also found that the pyramidal antenna has good characteristics on all three criteria given its limited aperture size. 
Table 2: Description of the simulated radiation patterns and the abbreviations used for them, as well as the mean values (averaged over all frequencies and all turbulence levels from $0.25 \%$ to $10 \%$ ) of the antenna coupling (AC) (percentage of power returning to the antenna), the fraction of invalid runs (IR) and the fraction of AC caused by rays undergoing a trajectory with multi-reflections (MR).

\begin{tabular}{|c|c|c|c|c|}
\hline Description & Abbrv. & AC & IR & MR \\
\hline $\begin{array}{l}\text { pyramidal aperture antenna with } D=14 \mathrm{~mm} \\
\text { in the poloidal plane }\end{array}$ & PYR & $4.46 \%$ & $7.04 \%$ & $14.32 \%$ \\
\hline Fundamental cylindrical Gaussian radiation patterns & & & & \\
\hline$w_{0}=5.88 \mathrm{~mm}$ and $D=14 \mathrm{~mm}$ & G14F & $4.68 \%$ & $3.26 \%$ & $32.71 \%$ \\
\hline$w_{0}=0.588 \lambda$ and $D=14 \mathrm{~mm}$ & G14L & $3.9 \%$ & $6.4 \%$ & $39.49 \%$ \\
\hline$w_{0}=\lambda$ and $D=24 \mathrm{~mm}$ & G24L & $5.53 \%$ & $3.29 \%$ & $25.18 \%$ \\
\hline$w_{0}=12 \mathrm{~mm}$ and $D=28 \mathrm{~mm}$ & $\mathrm{G} 28 \mathrm{~F}$ & $6.65 \%$ & $4.16 \%$ & $6.29 \%$ \\
\hline$w_{0}=1.2 \lambda$ and $D=28 \mathrm{~mm}$ & G28L & $6.00 \%$ & $3.46 \%$ & $18.35 \%$ \\
\hline $\begin{array}{l}\text { Superpositions of TEM } \\
\text { modes with } w_{0}=0.588 \lambda \text { and } T E M_{10}\left(\text { relative phase } 180^{\circ}\right) \\
\text { mom }\end{array}$ & & & & \\
\hline $90 \% \mathrm{TEM}_{00}$ and $10 \% \mathrm{TEM}_{10}$ & S9010 & $2.12 \%$ & $8.74 \%$ & $36.77 \%$ \\
\hline $80 \% \mathrm{TEM}_{00}$ and $20 \% \mathrm{TEM}_{10}$ & S8020 & $2.17 \%$ & $8.44 \%$ & $38.53 \%$ \\
\hline $70 \% \mathrm{TEM}_{00}$ and $30 \% \mathrm{TEM}_{10}$ & S7030 & $2.18 \%$ & $8.46 \%$ & $39.45 \%$ \\
\hline $60 \% \mathrm{TEM}_{00}$ and $40 \% \mathrm{TEM}_{10}$ & S6040 & $2.17 \%$ & $9.15 \%$ & $39.20 \%$ \\
\hline $50 \% \mathrm{TEM}_{00}$ and $50 \% \mathrm{TEM}_{10}$ & $\mathrm{~S} 5050$ & $2.06 \%$ & $8.98 \%$ & $39.88 \%$ \\
\hline
\end{tabular}

\section{Radiation pattern choice}

Considering that the tolerances for the installation of the components in ITER are expected to be in the order of a few mm [19], the radiation pattern G14L with $w_{0}(\lambda)=0.588 \lambda$ and $D=14 \mathrm{~mm}$ seems the best option for a frequency-independent PPR antenna for ITER. If more space was available, a more performant antenna with larger aperture and fundamental Gaussian radiation patterns could be used.

A direct comparison of the simulation results of G14L and those of the PYR radiation pattern, which was used in the current ITER PPR design (but which is strongly frequency dependent and therefore less suited for advanced interpretative models), the Gaussian has better performance when it comes to number of invalid runs, especially for high amounts of turbulence (at $10 \%$ turbulence on average $9 \%$ invalid runs against $19 \%$ for PYR). Both radiation patterns have similar antenna coupling at low frequencies, but PYR has better antenna coupling at higher frequencies. It is an inevitable consequence of a frequency-independent antenna that it has a broader main lobe than a frequency-dependent antenna at high frequencies, which causes this difference. Because of the broader main lobe of the Gaussian, the returning power of G14L is more often from indirect trajectories (with parasitic wall reflections) rather than from unperturbed trajectories. As a consequence the share of power reaching the receiver through imperfect (multi-reflection) paths is significantly higher. 


\section{Antenna design}

\subsection{Methodology}

To find an antenna shape which results in the G14L radiation pattern, an optimization is done using PROFUSION [32], a Linux commandline code package containing numerous tools to calculate microwave beam phenomena. A cylindrical antenna optimizer, based on a combination of simulation annealing and a downhill simplex method [33], is available and provides the possibility to optimize an antenna shape to resemble aperture fields as close as possible. The optimizer is used with aperture fields according to $w_{0}=0.588 \lambda$ at frequencies $30,37.5,45,52.5$ and $60 \mathrm{GHz}$ as optimization goal. Additional geometrical parameters need to be given to the optimizer: $D_{1}$, the input diameter of the antenna at the waveguide end; $D_{\max }$, the maximum aperture diameter (also limiting the diameter that the antenna can have at any location apart from the aperture); $l$ and $\Delta l$, the nominal length of the antenna and the tolerated deviation from it; $\theta_{\max }$ and $\theta_{\min }$, an upper and lower boundary for the slope of the geometry function $r(z)$ which describes the antenna shape.

\subsection{Optimization procedure}

A lower limit to $D_{1}$ is given by the cut-off frequency of the waveguide that feeds the antenna. $\mathrm{TE}_{m n^{-}}$ and $\mathrm{TM}_{m n}$ - eigenmodes in a smooth-walled cylindrical waveguide with radius $a$ have the following cut-off frequency:

$$
\begin{aligned}
f_{c}^{\mathrm{TM}} & =\frac{c j_{m n}}{2 \pi a} \\
f_{c}^{\mathrm{TE}} & =\frac{c j_{m n}^{\prime}}{2 \pi a}
\end{aligned}
$$

With $j_{m n}$ the $n^{\text {th }}$ zero of the $m^{\text {th }}$ Bessel-function and $j_{m n}{ }_{m n}$ the $n^{\text {th }}$ zero of the first derivative of the $m^{\text {th }}$ Bessel-function. Equation 4.1a gives that a waveguide with $D=6 \mathrm{~mm}$ has a TE 11 cut-off frequency $f_{c}=$ $29.28 \mathrm{GHz}$, meaning that the frequencies in range $f \in[30 \mathrm{GHz}, 60 \mathrm{GHz}]>f_{c}$ can propagate through it. Taking a margin on this minimum leads to $D_{1}=8 \mathrm{~mm}$. Initially the other parameters were chosen to match the poloidal size of the pyramidal reference antenna used in [19]: $l=115 \mathrm{~mm}$, with $\Delta l=10 \mathrm{~mm}$, $D_{\max }=14 \mathrm{~mm}$ and $\theta_{\max }=90^{\circ}$ and $\theta_{\min }=0^{\circ}$ (strictly diverging antenna). These initial parameters did not give a satisfying result: optimizations were finding an optimum with too much radiation in unwanted regions that showed frequency-dependent characteristics for at least a part of the $30-60$ $\mathrm{GHz}$ octave. By loosening the design constraints to $\Delta l=70 \mathrm{~mm}, \theta_{\min }=-60^{\circ}$ and $D_{\max }=20 \mathrm{~mm}$ a good optimization was found, having on average only $1.67 \%$ of its power in modes different from the frequency-independent optimization goal. The resulting optimal antenna shape is seen in Figure $3 \mathrm{a}$ and a possible 3D realization in Figure 3b.

The antenna shape is not monotonically flared outwards, but has two diverging sections with a converging section in between. Also $D=19.598 \mathrm{~mm}>14 \mathrm{~mm}$ is not ideal, since it is not sure that the antenna will easily fit in between the blanket interspace. This antenna would still fit in the provided space given a mounting precision of $2 \mathrm{~mm}$ for both blankets and antenna. The optimized antenna has $\operatorname{MAX}\left(w_{0}\right) / D=0.3$. Under the assumption that the required frequency-independent optimization can always be done with this ratio, it would be possible to make an antenna with $w_{0}(\lambda)=0.42 \lambda$ for $D=14$ $\mathrm{mm}$. This type of antenna would however have a broader pattern with 


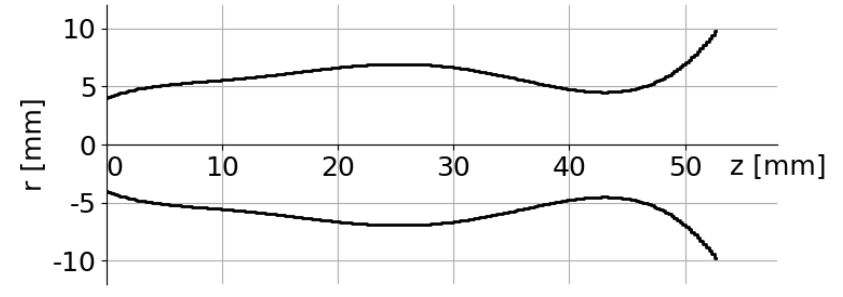

(a)

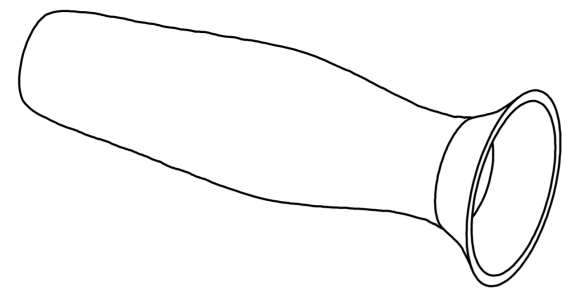

(b)

Figure 3: (a) Optimized antenna shape with $D_{\text {in }}=8 \mathrm{~mm}, D=19.598 \mathrm{~mm}, l=52.678 \mathrm{~mm}$, (b) Trimetric view of a possible realization of the optimized antenna. The antenna has on average $98.33 \%$ overlap with the optimization goal i.e. the fundamental Gaussian patterns with $w_{0}=$ $0.588 \lambda$.

more power radiated in the unwanted $|\theta|>0.7$ rad region. It is preferable to keep the slightly larger aperture antenna with $w_{0}=0.588 \lambda$.

\subsection{Higher-order mode analysis}

Because of the choice for $D_{1}=8 \mathrm{~mm}$, some higher-order modes can exist in the antenna input. In Table 3 the cut-off frequencies for a waveguide of this size are given. Not only the fundamental $\mathrm{TE}_{11}$, but also $\mathrm{TM}_{01}$ exists for the whole frequency band of 30 to $60 \mathrm{GHz}$ and $\mathrm{TE}_{21}$ exists for almost the whole frequency band. When the antenna input field contains one of these modes, this might lead to changes in the resulting radiation pattern. One of the things which can change is the direction of the main lobe, which can have large influence on the multi-reflections and antenna performance. PROFUSION simulations were done with $1 \%$ higher-order modes at the antenna input. For none of the frequencies did $1 \% \mathrm{TM}_{01}$ result in any significant angular offset in the poloidal direction. However, $1 \% \mathrm{TE}_{21}$ resulted in sometimes large offsets of up to $1.54^{\circ}$ at $37.5 \mathrm{GHz}$ (when there is $180^{\circ}$ relative phase between $\mathrm{TE}_{01}$ and $\mathrm{TE}_{21}$ ) and $1.24^{\circ}$ at $45 \mathrm{GHz}$ (with $270^{\circ}$ relative phase). At other frequencies the offsets were limited to less than $1^{\circ}$. Care must be taken in the design of the transmission line to the PPR antennas to avoid these higher-order modes, which is challenging considering that the oversized waveguides leading to the PPR antennas in ITER will be very long and involve a lot of bends [34]. If higher-order modes at the antenna input are inevitable, another option would be to make a new antenna optimization which uses the real antenna input. For this, a mode analysis of the transmission line should be done first. 
Table 3: Cut-off frequencies in a smooth-wall cylindrical waveguide with $D=8 \mathrm{~mm}$. Two modes can propagate for the whole bandwidth of 30 to $60 \mathrm{GHz}$, four more modes can propagate for the part of the bandwidth with $f>f_{c}$.

\begin{tabular}{|r|cccccc|cc|}
\hline mode & $\mathrm{TE}_{11}$ & $\mathrm{TM}_{01}$ & $\mathrm{TE}_{21}$ & $\mathrm{TE}_{01}$ & $\mathrm{TM}_{11}$ & $\mathrm{TE}_{31}$ & $\mathrm{TM}_{21}$ & $\mathrm{TE}_{41}$ \\
\hline$f_{c}(\mathrm{GHz})$ & 21.96 & 28.69 & 36.43 & 45.71 & 45.71 & 50.11 & 61.26 & 63.43 \\
\hline
\end{tabular}

\section{Experimental prototype testing}

Because the PROFUSION optimization is done on the antenna aperture fields, the cylindrical antenna optimization tool does not allow external structures such as blankets to be modelled during the optimization. This limitation means that the optimized antenna might suffer from similar effects as described by Valera et al for square aperture antennas [19]: apart from introducing multi-reflections, the blankets can also change antenna radiation patterns. To assess the performance of the antenna in different surroundings, a prototype of the optimized antenna, and a conical reference antenna with identical dimensions (length $l=52.678 \mathrm{~mm}$, input and output diameter $D_{\text {in }}=8 \mathrm{~mm}$ and $D=19.598$ $\mathrm{mm}$ ) were built and radiation pattern measurements were made for both antennas with and without mock-up blankets.

\subsection{Measurement setup}

The antennas were milled out of a $28 \mathrm{~mm}$ diameter aluminium cylinder, leaving the outer diameter at $28 \mathrm{~mm}$ to reduce the production cost and simplify mounting the antennas in a measurement setup. A mock-up version of the ITER blanket structure was made from aluminium. The blanket geometry is prescribed by Figure 1 in the poloidal plane and the blankets are $153 \mathrm{~mm}$ long in toroidal direction, which is the same as used by Belo et al in [20]. To guarantee that the distance between the blankets is $2 h=30 \mathrm{~mm}$, two cylindrical spacers are placed between the blankets. The CAD-design of the antenna mounted in the blanket structure is illustrated in Figure 4a. Also depicted at this figure is the transition from the antenna input diameter $D_{\text {in }}=8 \mathrm{~mm}$ to a standard WR-28 waveguide, so that the antenna can easily be connected to standard components.

Measurements at 30,37.50, 45, 52.50, and $60 \mathrm{GHz}$ are done. The two lowest frequencies are located in the Ka-band $(26.5-40 \mathrm{GHz})$, the others fall in the V-band $(40-75 \mathrm{GHz})$. Since the V-band frequencies can propagate in Ka-band waveguides, a single transition piece from a cylindrical waveguide with diameter $8 \mathrm{~mm}$ to a WR-28 Ka-band waveguide can be used for all measurements. For the V-band measurements an extra transition follows on this from WR-28 to WR-15, the V-band standard waveguide. The measurement accuracy of the network analyzer is

$$
\max \left(0.2 \mathrm{~dB}, 0.01 S_{\mathrm{dB}}\right)
$$

with $S_{\mathrm{dB}}$ the measured signal amplitude. Although measurements at $45 \mathrm{GHz}$ can be done with the Vband equipment, their accuracy might be lower than given by this expression, as the used network analyzer specifies the V-band only for the range $50-75 \mathrm{GHz}$.

The test-antenna (with or without blankets) is used as emitter, while an open ended WR-28 waveguide, functioning as receiver, rotates around it and registers both the amplitude and phase of the electric field. A picture of the setup is given in Figure $4 \mathrm{~b}$. All metallic objects in the direct 


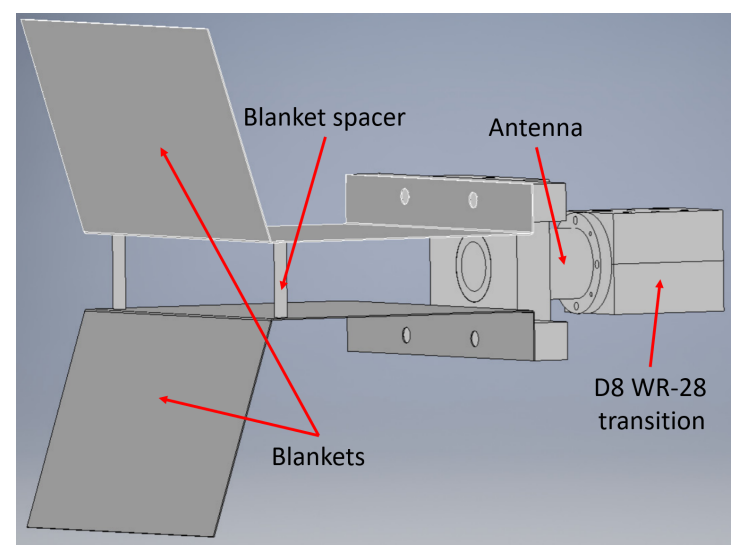

(a)

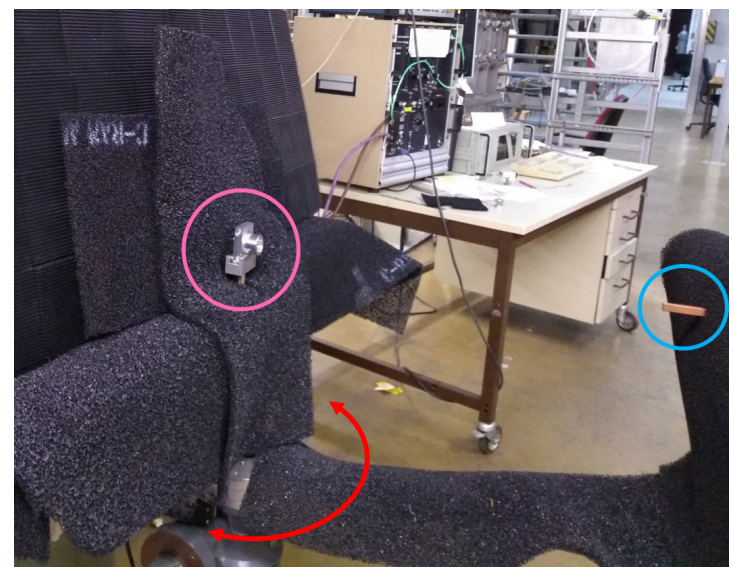

(b)

Figure 4: (a) Assembly drawing of a prototype antenna surrounded by a blanket structure, (b) laboratory setup with the test-antenna (pink circle) mounted on pivot point and the receiver (blue circle) mounted on a rotating arm.

environment of the measurements are covered with microwave absorbing material (black on Fig. $4 \mathrm{~b})$ to minimize their effects on the measurements. The distance between the transmitting and receiving antenna was $50 \mathrm{~cm}$. With the far-field starting at $16 \mathrm{~cm}$ (most strict condition from $2 \frac{D^{2}}{\lambda}$, $5 D$ and $1.6 \lambda$ evaluated for all frequencies $[35,36])$, the measurements are taken in the Fraunhofer region.

\subsection{Radiation diagram analysis}

The results of the comparison between reference and optimized antenna are presented in Figures 5 and 6 . The radiation patterns are normalized to unity power in the poloidal plane using

$$
W_{\text {normalized }}(\theta)=\frac{W(\theta)}{\int_{-90^{\circ}}^{+90^{\circ}}}
$$

$W$

In Figure 5a-b, the results of the optimization can be seen: the directivity and shape of the optimized antenna diagrams are a lot more homogeneous than for the conical antenna, whose diagram gets narrower and shows a strong increase in directivity when the frequency rises. When the blankets are added to the setup, the directivity of the optimized antenna remains less frequency dependent than that of the conical antenna. However, the shape of the optimized antenna diagrams is no longer frequency independent, but shows strong variations depending on the frequency, with sidelobes at different locations for all frequencies and a double-hump shape appearing at $45 \mathrm{GHz}$.

In Figure 6, the same information is presented in polar coordinates and a logarithmic scale. The radiation diagram of the conical antenna shows a better directivity than the optimized antenna, both with and without blankets. The difference between the directivity of the antennas varies depending on the frequency. Also here it can be seen that adding the blankets to the setup undoes part of the optimization done by creating frequency dependence and high sidelobes.

The influence of the blankets on the radiation diagram makes it no longer frequency-independent, so that it does not, as intended, reduce the complexity of the problem. The change in radiation 


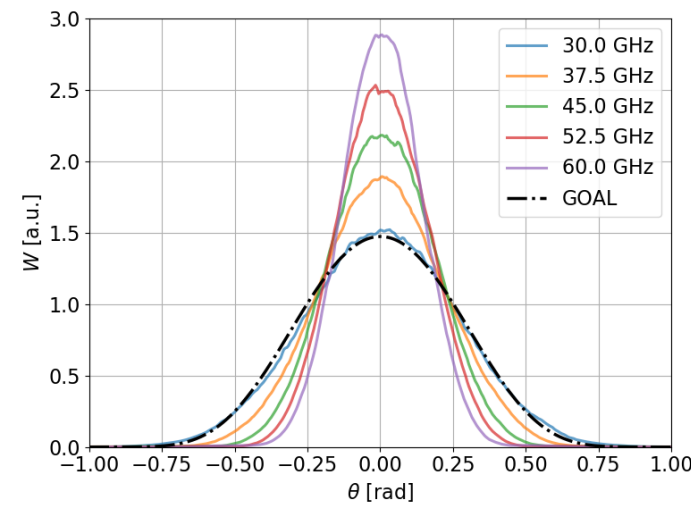

(a) Conical reference antenna (no blankets)

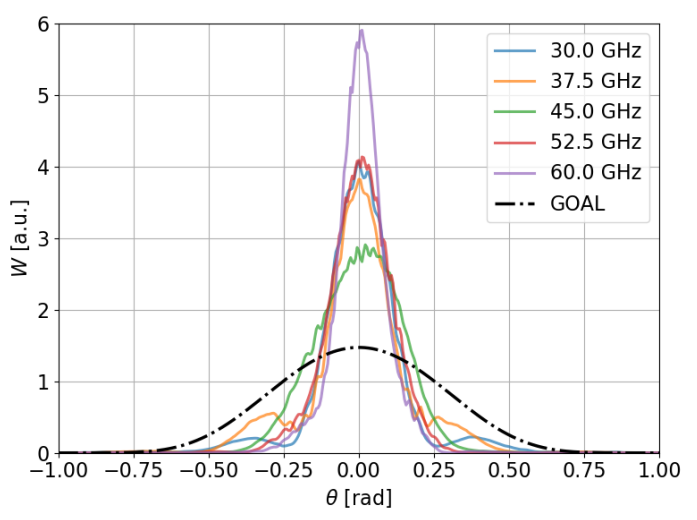

(c) Conical reference antenna with blankets

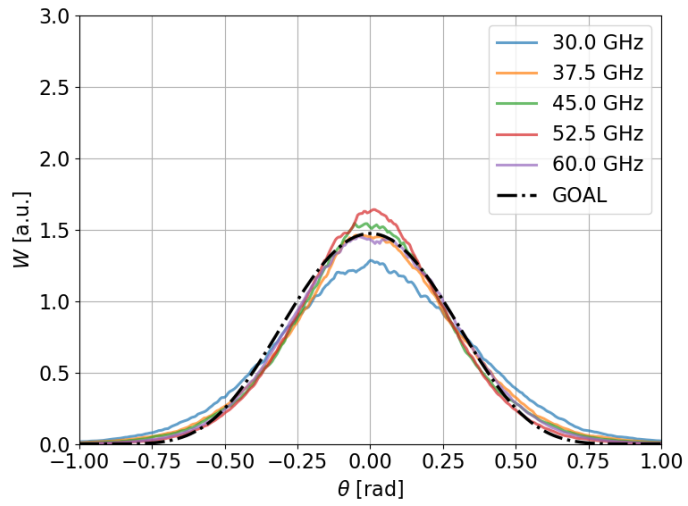

(b) Optimized antenna (no blankets)

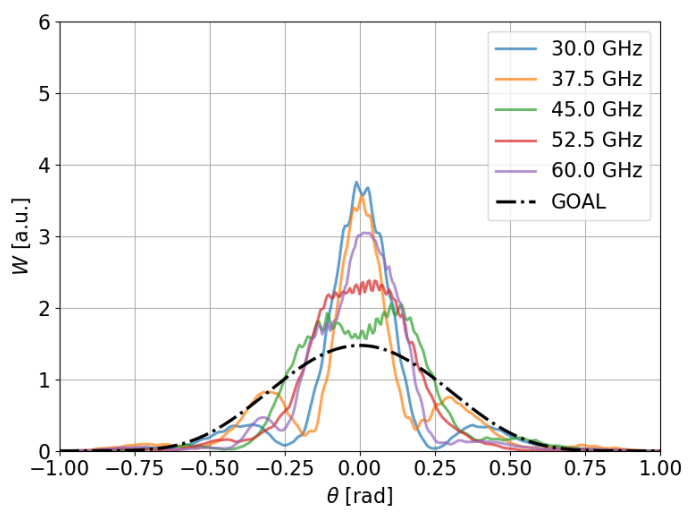

(d) Optimized antenna with blankets

Figure 5: Linear-scaled poloidal (H-plane) radiation diagrams of the prototype antennas. The optimization goal, $\mathrm{TEM}_{00}$ with $w_{0}=0.588 \lambda(D=14 \mathrm{~mm})$ is drawn for comparison.

diagram also means that the performance of the antenna, which was assessed with the ray tracing code, has changed. The sidelobes, which do not show when the antenna is used without blanket environment but are present when mounted in the environment, can cause unwanted direct reflections to the receiver, lowering the performance of the antennas. In addition to these measurements, different versions of the mock-up blankets with different lengths $L$ (the length of the narrow part between the blankets, see Figure 1) were made to evaluate how the length of this section influences the radiation diagram. In addition to the blankets with $L=120 \mathrm{~mm}$

(which are used in other studies, ray tracing simulations, and the measurements presented Fig. 5 and 6 ) blankets with $L=30,60$ and $90 \mathrm{~mm}$ were used. The results can be seen in Figure 7 for 30 and 52.5 GHz. It is seen that for $30 \mathrm{GHz}$ the blankets with $L=60 \mathrm{~mm}$ result in an antenna diagram which is close to the optimization goal. However, at other frequencies such as $52.5 \mathrm{GHz}$, this is not the case. Based on these results, there is no preferred value of $L$. However, already for the currently ${ }_{220}$ used $L=$ $120 \mathrm{~mm}$ the heat loads on the antennas could be problematic [37], so $L<120 \mathrm{~mm}$ is not 


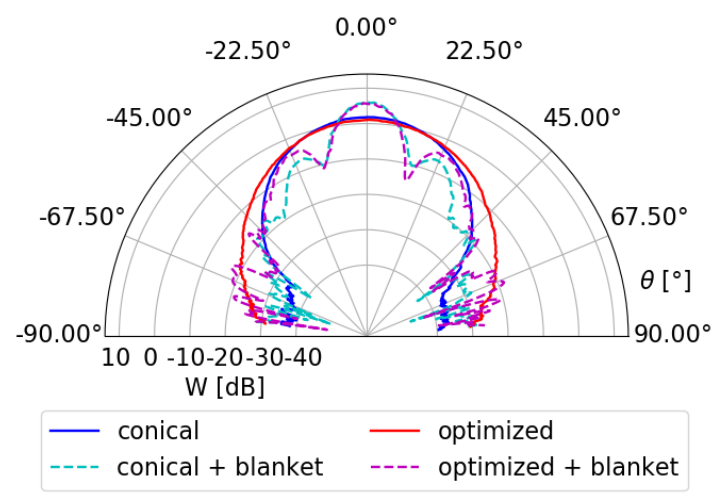

(a) $30 \mathrm{GHz}$

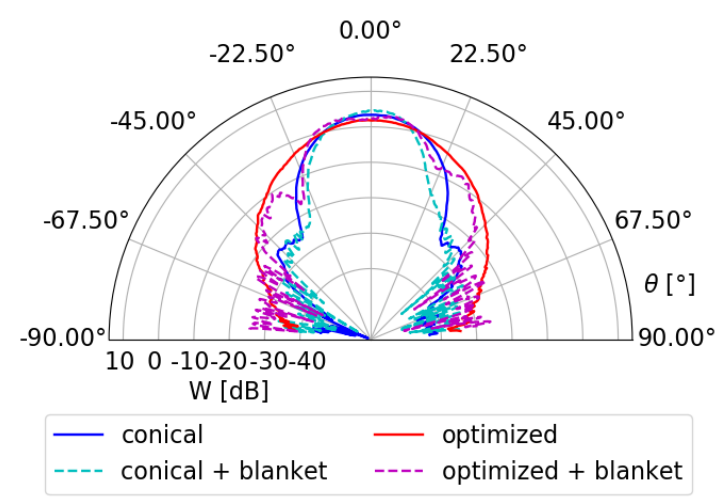

(c) $45 \mathrm{GHz}$

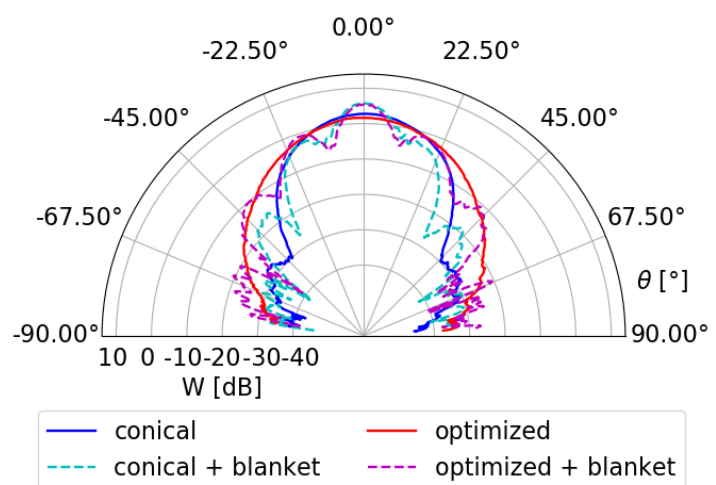

(b) $37.50 \mathrm{GHz}$

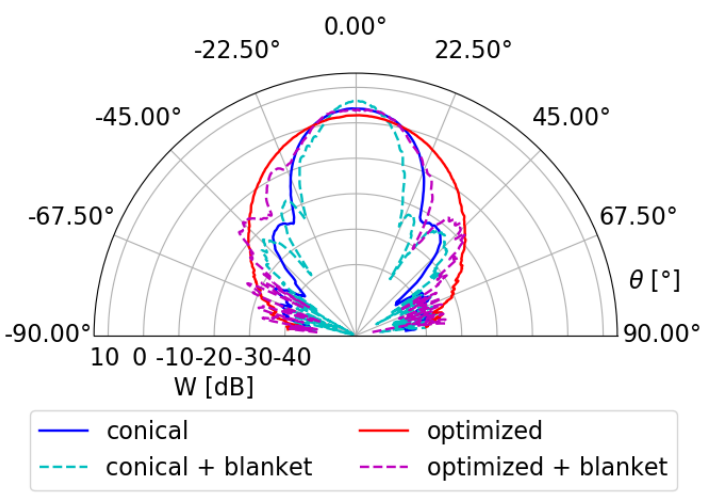

(d) $52.50 \mathrm{GHz}$

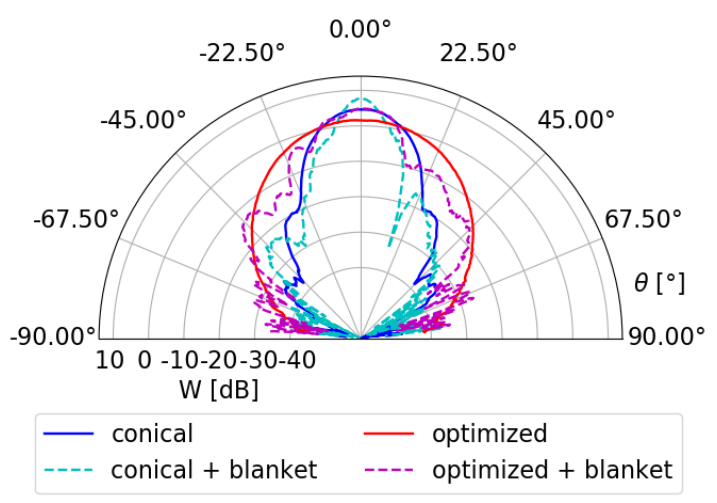

(e) $60 \mathrm{GHz}$

Figure 6: Poloidal (H-plane) radiation diagrams of the optimized and reference conical antenna prototypes with and without blankets at different frequencies. The reference antenna has a higher directivity for all frequencies. The blankets cause sidelobes and a change in directivity and radiation shape for both antennas.

advisable from a thermal point of view.

In Figure 7, multiple radiation patterns are asymmetrical. Misalignment is a known problem 


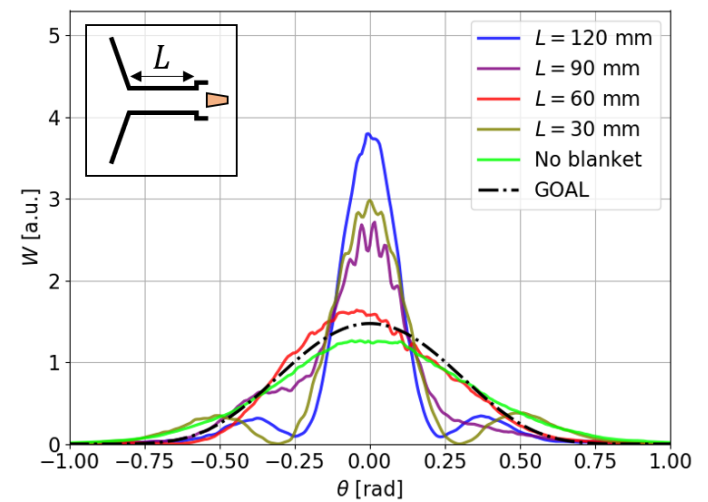

(a) $30 \mathrm{GHz}$

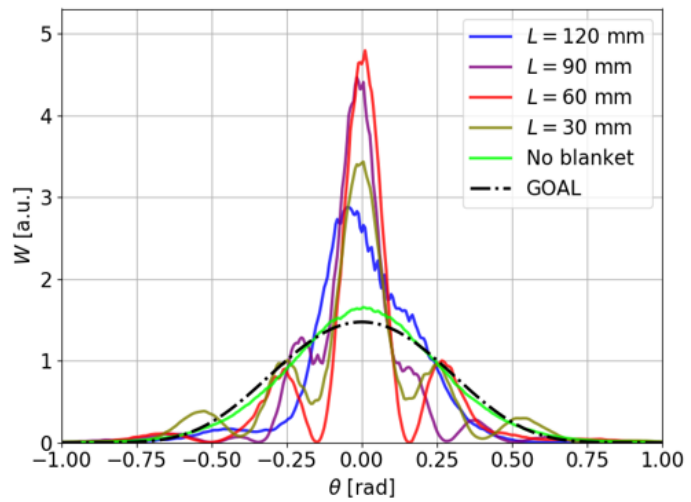

(b) $52.5 \mathrm{GHz}$

Figure 7: Linear-scaled poloidal radiation diagrams of the optimized prototype antenna when using blanket structures with different lengths $L$. The optimization goal, $\mathrm{TEM}_{00}$ with $w_{0}=0.588 \lambda$ $(D=14 \mathrm{~mm})$ is drawn for comparison.

of reflectometers [38] and the asymmetry observed can be attributed to misalignment between the antenna and blanket structure, which can be introduced with each mechanical change done on the setup in between measurements. This explanation is supported by comparison of Figure $7 \mathrm{~b}$ with Fig. 5d, which also shows results for the $L=120 \mathrm{~mm}$ measurement at $52.5 \mathrm{GHz}$, but measured in a measurement sequence with few mechanical changes. For the identical measurements in Fig. $5 \mathrm{~d}$ the asymmetry is not present. The observed asymmetry indicates that correct alignment of the setup is crucial for the resulting radiation diagram. Not only does this put strict tolerances on the antenna mounting, it also emphasizes the importance of the mode analysis of the antenna input (Section 4). High mode purity at the antenna input is required as small angular offsets (which can be caused by higher-order modes) could result in large asymmetry when taking the blankets into account. Additionally, an extended study on the thermal behaviour of the complete system should be made to evaluate the effect of possible thermal expansion (resulting in e.g. a reduction of the blanket interspace or a change in the antenna position) on the radiation patterns.

\section{Conclusions}

With ray tracing simulations in the poloidal plane, different antennas were compared on their performance as plasma positioning reflectometry (PPR) antenna, using the ITER gap 6 PPR environment with a forward scattering turbulence model similar to the one used in [27]. Independent of the radiation pattern and probing frequency, the antennas showed a maximum antenna coupling at $3 \%$ turbulence, agreeing with the Doppler reflectometry full-wave simulation results of da Silva et al ${ }_{342}$ [27] done in similar conditions.

For antennas with fundamental Gaussian radiation patterns, it was found that increasing the antenna aperture and beam waist significantly increases the antenna coupling and PPR performance. Providing space for larger PPR antennas would therefore result in an increasing performance. When taking the current ITER gap 6 spatial limitations as fixed, the pyramidal horn antenna (PYR) studied 
by P. Valera, J.H. Belo and A. Silva [19, 20] performs very well. From the simulated patterns, the antenna with beam waist/wavelength relation $w_{0}=0.588 \lambda$ and diameter $D=14 \mathrm{~mm}(\mathrm{G} 14 \mathrm{~L})$ has the best performance when aiming for frequency-independent antenna characteristics while respecting the spatial constraints.

Based on the G14L radiation pattern, the PROFUSION cylindrical horn antenna shape op-352 timizer [33] was used to design an antenna with frequency-independent characteristics in the ${ }_{353} 30-60 \mathrm{GHz}$ octave, relevant for O-mode reflectometry.

Prototype testing with the optimally-shaped horn antenna and a conical reference antenna proved the optimized antenna to have frequency-independent emission characteristics. However, withing an ITER blanket mock-up environment, the changes in the antenna near field caused the optimization effects to disappear. Changing the antenna position relative to the blankets (corresponding to moving it closer to the plasma) did not result better frequency-independent radiation patterns. Multiple measurements show that small misalignment of the antenna in the blanket environment causes strong asymmetry in the radiation patterns, indicating the need of ${ }_{361}$ careful mounting and a further study on possible thermal expansion [37], which could introduce asymmetry in the setup during tokamak operation. Additional PROFUSION simulations with the optimally-shaped antenna showed that also small fractions of higher-order modes at the antenna feed result in asymmetry in the radiation pattern. Considering the length and many bends [34] of the ITER waveguide connecting the antenna, care should be taken in the final antenna and waveguide design to minimize these effects.

Building further on the research presented here, a next step is to find an optimal shape of the antenna, so that it has frequency-independent behaviour taking into consideration the blanket environment. This optimization could e.g. be performed with HFSS [30]. The use of frequency-independent antennas could then lead to lower computational complexity of PPR interpretative models able to extract both density profile and density fluctuation information from the measurements.

\section{References}

[1] R. Sabot, F. Clairet, G. Conway, J. Giacalone, T. Gerbaud, S. Hacquin et al., Recent results on turbulence and MHD activity achieved by reflectometry, Plasma Phys. Control. Fusion 48 (2006) .

[2] L. Vermare, F. Clairet, S. Heuraux and G. Leclert, Rational surfaces localization and MHD activity measurements using fast sweep reflectometry, Plasma Phys. Control. Fusion 47 (2005) .

[3] H. Arnichand, R. Sabot, S. Hacquin, A. Krämer-Flecken, X. Garbet and J. Citrin, Quasi-coherent modes and electron-driven turbulence, Nucl. Fusion 54 (2014) .

[4] T. Estrada, K. Nagasaki, E. Blanco, G. Perez and V. Tribaldos, Microwave Reflectometry Diagnostics: Present Day Systems and Challenges for Future Devices, Plasma and Fusion Res. 7 (2012) .

[5] D. K. A. Mase, Y. Kogi et al., Development and application of radar reflectometer using micro to infrared waves, Adv. Phys. 3 (2018).

[6] F. Clairet, C. Bottereau, A. Medvedeva et al., 1 us broadband frequency sweeping reflectometry for plasma density and fluctuation profile measurements, Rev. Sci. Instrum. 88 (2017) .

[7] L. Cupido, S. Graca, G. Conway, M.Manso, F.Serra and A. U. Team, Frequency hopping millimeter-wave reflectometry in ASDEX upgrade, Rev. Sci. Instrum. 77 (2006) . 
[8] G. Conway, J. Schirmer, S. Klenge, W. Suttrop, E. Holzhauer and A. U. Team, Plasma rotation profile measurements using Doppler reflectometry, Plasma Phys. Control. Fusion 46 (2004) .

[9] E. Ricardo, F. da Silva, S. Heuraux and A. Silva, Assessment of a multi-reflectometers positioning system for DEMO plasmas, J. Instrum. 14 (2019) .

[10] G. Kramer, E. Valeo, A. Zolfaghari and N. Bertelli, Antenna-plasma coupling calculations for the ITER low-field side reflectometer, Nucl. Fusion 58 (2018) .

[11] C. Barès, C. Brousseau, L. L. Coq and A. Bourdillon, Effect of Antenna Phase Center Displacement on FM CW Measurements â Application to Radar System, Electronics Letters 39 (2003) .

[12] R. B. Morales, S. Hacquin, S. Heuraux and R. Sabot, New density profile reconstruction methods for X-mode reflectometry, Rev. Sci. Instrum $\mathbf{8 8}$ (2017).

[13] R. Morales, S. Heuraux, R. Sabot and S. Hacquin, The reconstruction of hollow areas in the density profile from frequency swept reflectometry, Plasma Science Techno. 22 (2020) .

[14] T. Gerbaud et al., Comparison of density fluctuation measurements between O-mode and X-mode reflectometry on Tore Supra, Rev. Sci. Instrum. 77 (2006) .

[15] J. Santos et al., Reflectometry-based plasma position feedback control demonstration at ASDEX upgrade, Nucl. Fusion 52 (2012) .

[16] A. Silva et al., Microwave reflectometry diagnostic for density profile and fluctuation measurements on ASDEX upgrade, Rev. Sci. Instrum. 70 (1998).

[17] F. da Silva, S. Heuraux, E. Ricardo, P. Quental and J. Ferreira, Assessment of the measurement performance of the in-vessel system of gap 6 of the ITER plasma position reflectometer using a finite-difference time-domain Maxwell full-wave code, Rev. Sci. Instrum. 87 (2016) .

[18] F. da Silva, S. Heuraux and M. Manso, Developments on reflectometry simulations for fusion plasmas: application to ITER position reflectometers, J. Plasma Phys. 72 (2006) .

[19] P. Valera, A. Silva and J. Belo, Testing of the ITER plasma position reflectometry high-field side in-vessel antenna assembly prototype, Rev. Sci. Instrum. 89 (2018) .

[20] J. Belo, P. Valera and A. Silva, Benchmarking of the EM modelling of the ITER plasma position reflectometry in-vessel antennas with a metallic target using prototype tests, J. Instrum. 15 (2020) .

[21] A. Silva, F. da Silva, S. Heuraux and B. Gonçalves, First assessment of microwave diagnostics for DEMO, Fusion Eng. Des. 96 (2015) .

[22] E. Tracy, A. Brizard, A. Richardson and A. Kaufman, Ray Tracing and Beyond. Cabridge University Press, 2014.

[23] C. Honoré, P. Hennequin, T. A. and A. Quéméneur, Quasi-optical gaussian beam tracing to evaluate doppler back-scattering conditions, Nucl. Fusion 46 (2006) .

[24] B. Chaudhury and S. Chaturvedi, Comparison of wave propagation studies in plasmas using three-dimensional finite-difference time-domain and ray-tracing methods, Phys. Plasmas 13 (2006) .

[25] C. Fanack et al., Ordinary-mode reflectometry: modification of the scattering and cut-off responses due to the shape of localized density fluctuations, Plasma Phys. Control. Fusion 38 (1996) .

[26] E. Gusakov, A. Surkov and A. Popov, Multiple scattering effect in Doppler reflectometry, Plasma Phys. Control. Fusion 47 (2005) .

[27] F. da Silva, S. Heuraux, E. Gusakov and A. Popov, A numerical study of forward-and backscattering signatures on doppler-reflectometry signals, IEEE Plasma Sci. 38 (2010) . 
[28] U. Stroth, Plasmaphysik. Vieweg + Teubner Verlag Springer Fachmedien, 2011.

[29] C. Fanack, "Etude analytique et numérique de la réflectométrie dans un plasma fluctuant: modèles à une et deux dimensions." 1997.

[30] H. Hugon, "Study and optimisation of the antennas for the ITER plasma-position reflectometry diagnostics system." 2014.

[31] E. Sysoeva, F. da Silva, E. Gusakov, S. Heuraux and A. Popov, Electron cyclotron resonance heating beam broadening in the edge turbulent plasma of fusion machines, Nucl. Fusion 55 (2015) .

[32] B. Plaum, Simulation of microwave beams with profusion (2019 edition), OPUS - Online Publikationen der Universität Stuttgart (2019) .

[33] B. Plaum, Optimization of broadband smooth-wall circular horn antennas, J. Infrared Millim. Te. 39 (2018) .

[34] P. Valera, J. Belo, A. Silva and F. da Silva, Design status of the in-vessel subsystem of the ITER Plasma Position Reflectometry system, J. Instrum. 14 (2019) .

[35] C. Balanis, Antenna Theory: Analysis and Design. Wiley, 2016.

[36] W. Stutzman and G. Thiele, Antenna Theory and Design. Wiley, 2012.

[37] J. Nietiadia, C. Vidala, R. Luisa and P. Varela, Thermal analyses of the in-vessel frontends of the ITER plasma position reflectometry system, Fusion Eng. Des. 156 (2020) .

[38] G. Conway, Effects of reflectometer asymmetries on fluctuation measurements, Plasma Phys. Control. Fusion 41 (1999). 